Revisión de Tema

\title{
Valor de la microcirugía como tratamiento de la infertilidad masculina en la era de la reproducción asistida
}

\author{
Héctor Narváez $\boldsymbol{R}^{*}$ *
}

Recibido: septiembre 17/2001 - Revisado: noviembre 20/2001 - Aceptado: mayo 30/2002

\begin{abstract}
RESUMEN
La actuación sobre el tubo epididimario y el conducto deferente obstruidos con diámetros aproximados de 0.1 y $0.3 \mathrm{~mm}$ respectivamente, precisan de la aplicación de técnicas microquirúrgicas. La microcirugía también puede hallarse indicada en determinados casos de varicocelectomía.

Por encima de todos los tópicos que se apuntan con relación a la microcirugía, cabe mencionar que los factores más importantes son la práctica y la experiencia, por lo que es conveniente tener la oportunidad de realizar estas cirugías de manera frecuente en la clínica humana.

Palabras clave: microcirugía, epididimovasostomía, vasovasostomía, varicocelectomía, fertilización in vitro, inyección espermática intracitoplasmática.
\end{abstract}

\section{SUMMARY}

The action over the epidimary tube and the deferent blocked conduct with diameter of approx. 0.1 and $0.3 \mathrm{~mm}$ respectively, specifies the applications of the microsurgery techniques. The microsurgery may also be needed in some varicocelectomy cases.

Over all the subjects and topics related to the microsurgery, its necessary to mention that the most important factors are the practice and experience and though it is very convenient to carry out these surgeries frequently in the human clinic.

Key words: Microsurgery, epididimovasostomy, vasovasostomy, varicocelectomy, fertilization in vitro, intracitoplasmatic sperm injection.

\section{INTRODUCCIÓN}

El tratamiento microquirúrgico de la infertilidad masculina se limita fundamentalmente a la cirugía reconstructiva o desobstructiva de la vía seminal, a la recuperación quirúrgica de espermatozoides para utilizar en técnicas de reproducción asistida y a la cirugía del varicocele.

La vía seminal puede obstruirse a cualquier nivel, desde su segmento intratesticular hasta el conducto eyaculador, de forma total o parcial, uni o bilateralmente, a uno o varios niveles. Aunque la forma más observada en la clínica de infertilidad es la completa, bilateral y al mismo nivel dando lugar a azoospermia, también se pueden observar otras presentaciones, unilaterales o parciales en forma de oligozoospermia. No obstante, en muchas ocasiones no dan lugar a alteraciones seminológicas ni, lógicamente, a infertilidad.

En más del $90 \%$ de los casos, la obstrucción de la vía seminal es bilateral, completa y localizada a nivel del epidídimo (1). La vía seminal puede obstruirse por procesos inflamatorios, genéticos, traumáticos, neoplásicos, iatrogénicos, ser originada por un proceso intra o extraluminal, o bien por sección o lesión de En más del $90 \%$ de los casos, la obstrucción de la vía seminal es bilateral, completa y localizada a nivel del epidídimo (1). La vía seminal puede obstruirse por procesos inflamatorios, genéticos, traumáticos, neoplásicos, iatrogénicos, ser originada por un proceso

\footnotetext{
* Universidad Autónoma de Barcelona, Médico Andrólogo de Fecundar, Cali. Centro de Biomedicina ReproductivaFecundar. Carrera 40 No 5B-105 Tequendama, Cali, Valle. fecundar@colombianet.net
} 
intra o extraluminal, o bien por sección o lesión de la estructura tubular.

La anamnesis puede hacer sospechar una obstrucción de la vía seminal. Ejemplos de ello son las inflamaciones del contenido escrotal, cirugías previas escroto-in/guinales, uretro-prostato-vesiculitis, etc. No obstante, pueden no existir antecedentes significativos.

La exploración física puede evidenciar la ausencia de conductos deferentes, el aumento de tamaño y/o consistencia de los epidídimos, o el aumento de diámetro de los deferentes, tanto de uno como de ambos lados.

En el espermograma, una azoospermia puede ser manifestación de obstrucción bilateral. Un volumen seminal de $1 \mathrm{ml}$ es sugerente de ausencia de fluido vesicular, secundario a una obstrucción a la altura de los conductos eyaculadores o a una agenesia de la vía seminal. La presencia de leucocitos y/o hematíes puede ser indicativa de un proceso infeccioso, posible factor etiológico de la obstrucción (2).

En la biopsia testicular bilateral se evidencia una espermatogénesis sin alteraciones. Así mismo se observan las características macroscópicas del epidídimo.

La deferentovesiculografía (DVG) está indicada en el mismo acto de la biopsia testicular en aquellos casos donde exista una hipoespermia (volumen menor a $2 \mathrm{ml}$ ) con deferentes palpables (sospecha de obstrucción de los conductos eyaculadores) o en aquellos pacientes con antecedentes de posible obstrucción deferencial (cirugía inguinoescrotal, tuberculosis, etc.) (3).

En los casos de agenesia bilateral del conducto deferente, debe realizarse el estudio genético para valorar la posible presencia del gen de la fibrosis quística, localizado en el brazo largo del cromosoma 7 (4- 5).

$\mathrm{Al}$ plantear las diversas alternativas terapéuticas microquirúrgicas de la vía seminal, debe valorarse el tiempo de evolución del proceso, la etiología, la naturaleza congénita o adquirida, el nivel de obstrucción (uno o varios), la edad de la pareja, la disponibilidad de la pareja frente a las terapéuticas, así como las posibles patologías de la pareja.

Los importantes avances realizados en el campo de la reproducción asistida, y fundamentalmente en la fertilización in vitro (FIV), con la mayor efectividad de la inyección espermática intracitoplasmática (ICSI), han favorecido cambios notables en el pronóstico del hombre afectado de obstrucción de la vía seminal (6-8).

\section{CIRUGÍA DE LA VÍA SEMINAL}

El uso de magnificación y técnicas microquirúrgicas, es imprescindible en el manejo de las estructuras tubulares epididimarias y deferenciales con diámetros inferiores a $0.5 \mathrm{~mm}$. La dificultad de la práctica microquirúrgica es el tiempo que debe dedicarse a la misma en el laboratorio experimental durante el período de formación; posteriormente es necesario tener la oportunidad de aplicarla de forma constante en la clínica humana.

\section{Cirugía epididimaria}

Debido a que la epididimovasostomía es una cirugía relativamente larga y el epidídimo es un órgano considerablemente sensible, es aconsejable realizarla bajo anestesia general aunque, de hallarse indicado, también se puede practicar bajo anestesia local y sedación del paciente.

Sobre la cara anterior del escroto se realiza una incisión de 3 a $4 \mathrm{~cm}$ en la parte superior o raíz del mismo. Una vez expuesto el contenido escrotal se aprecian las características macroscópicas del testículo y del epidídimo.

Bajo el microscopio operatorio se realiza una hemisección transversal en la cara anterior deferencial hasta apreciar su luz. A través de la misma se comprueba la permeabilidad distal. A continuación se selecciona el área más distal epididimaria que presenta dilatación tubular. Con unas pinzas se levanta la túnica epididimaria y con las tijeras se hace una apertura circular en la misma, de diámetro aproximado al del conducto deferente. Se pasa un portaobjetos por la apertura tubular, mientras se observan las características macroscópicas del fluido que emana. Éste puede hallarse ausente, ser abundante o escaso, de aspecto claro o espeso y de color amarillo, blanco o transparente (10-11). Si no se constata la presencia de 
espermatozoides o éstos no son completos (sólo cabezas o colas cortadas) deben realizarse nuevas aperturas tubulares más proximales, incluso llegando a los conos eferentes hasta hallar espermatozoides completos.

El deferente es aproximado al tubo epididimario y se realizan 3 ó 4 puntos con nylon monofilamento de 9-0 entre la túnica epididimaria y la pared muscular deferencial posterior. Con el mismo sistema se realizan 2 ó 3 puntos con nylon monofilamento de 10-0 entre la pared posterior del tubo epididimario y la capa mucosa posterior de la luz deferencial. Una vez realizada la anastomosis posterior se precede a colocar 2 ó 3 puntos entre la mucosa deferencial y la pared anterior del túbulo epididimario (12).

$\mathrm{Al}$ mes de la intervención se realiza el primer espermograma y se repite con una periodicidad de un mes los primeros seis meses, y de dos meses hasta completar el año. A menudo los espermatozoides no aparecen en el eyaculado hasta transcurridos ocho meses. Los motivos de ello son difíciles de demostrar aunque parecen estar relacionados con el edema posquirúrgico y con una anastomosis poco precisa.

El mejor pronóstico se establece en aquellos pacientes en los cuales la obstrucción del epidídimo es de corto tiempo de evolución, localizada en la parte distal del órgano con espermatozoides abundantes y móviles en los que se ha practicado una correcta técnica quirúrgica. En los casos de etiología adquirida, generalmente inflamatoria o secundaria a una obstrucción más distal, se consiguen permeabilizaciones en el 63\% y gestaciones en el 34\% de los casos (13). Cuando la etiología es idiopática o congénita, el porcentaje de permeabilidad es de $37 \%$ y el de gestaciones de $19 \%$ (14-16).

\section{Cirugía deferencial}

Al referirse a la obstrucción del conducto deferente es muy importante diferenciar entre aquella que se establece después de haber realizado una vasectomía, y la que se produce por otras causas generalmente de tipo iatrogénico.

Entre el 2 y 6\% de hombres vasectomizados solicitan la posibilidad de recuperar la fertilidad, las causas de ello, en orden de frecuencia, son: cambio de pareja, muerte de hijos, deseo de nueva gestación en el seno de la misma pareja, vasectomía practicada sin tener ningún hijo e intolerancia psicológica a la esterilización (17).

El procedimiento se realiza bajo anestesia local. Se practica una incisión longitudinal de 2 a $3 \mathrm{~cm}$ a la altura de la raíz escrotal; se secciona transversalmente el deferente y se comprueba la permeabilidad distal. A continuación se secciona transversalmente el extremo deferencial preobstructivo y se observan las características macroscópicas del fluido (cantidad, color y aspecto) y en el mismo quirófano se valora la concentración y calidad espermática de acuerdo con la clasificación referida en la Tabla 1 (18-19).

Bajo visión microscópica se colocan tres puntos de nylon monofilamento de 10-0 en la capa mucosa de ambas caras anteriores deferenciales. Completa da la anastomosis muco-mucosa anterior se procede de igual forma en la cara posterior. Finalmente, se colocan de cuatro a seis puntos de nylon monofilamento de 9-0 entre ambas paredes musculares posterior y anterior, y se procede de igual forma en el escroto contralateral.

El primer espermograma se realiza al mes de la intervención. En principio los espermatozoides pueden ser escasos y de mala calidad para ir mejorando en los espermogramas sucesivos (uno por mes los primeros seis meses, y uno cada dos meses hasta completar el año). Los embarazos se presentan con mayor frecuencia entre los seis y doce meses posterior a la cirugía (20-21).

Cuando existe una buena concentración espermática con alteraciones de la motilidad, debe valorarse la presencia de anticuerpos antiespermáticos (AAE), y realizar un tratamiento con corticoesteroides si no hay ninguna contraindicación cuando se hallan presentes, o recurrir a técnicas de reproducción asistida (22).

Según lo reportado por Belker y colaboradores, los resultados del protocolo para realizar una vasovasostomía bajo anestesia local son: 


$\begin{array}{lcccc}\text { - Tiempo } & <3 & 3-8 & 9-14 & 15 \\ \text { vasectomia } & \text { afios } & \text { affos } & \text { affos } & 2 \text { afios } \\ \text { - Permeabilidad (\%) } & 97 & 88 & 79 & 71 \\ \text { - Gestacion (\%) } & 76 & 53 & 44 & 30\end{array}$

Estos resultados son superiores a los que se obtendrían mediante la microaspiración espermática y técnicas de reproducción asistida (23-24).

Cuando la lesión deferencial -de origen generalmente iatrogénico- se ha producido en un tiempo relativamente corto respecto al diagnóstico de la misma, es posible, en caso de hallar el extremo distal, practicar una vasovasostomía con la metodología ya descrita. No obstante, en la mayoría de casos el periodo de evolución es largo comportando la obstrucción secundaria del epidídimo, y el extremo deferencial distal es muy difícil de localizar (25). En otras ocasiones, si se localiza, la longitud del deferente no permite la correcta aproximación de ambos extremos para poder practicar la vasovasostomía. En estos últimos casos _así como en aquéllos en los que la obstrucción sea muy distal_ cabe considerar la posibilidad de proceder a una microaspiración espermática deferencial o epididimaria, o bien implantar un espermatocele artificial; esto es un reservorio de material sintético adherido al epidídimo previo al sitio de obstrucción para que se depositen los espermatozoides. Esta técnica fue abandonada por los malos resultados que se obtenían, fundamentalmente en los casos de agenesia de conductos deferentes (26).

Numerosos factores afectan los resultados de la vasovasostomía: la experiencia del cirujano, el tiempo de duración de la obstrucción y la técnica quirúrgica. Durante el acto quirúrgico, la calidad de fluido espermático obtenido del extremo testicular es predictivo del éxito posquirúrgico.

\section{Cirugía de los conductos eyaculadores}

Más del $80 \%$ de obstrucciones de los conductos eyaculadores cursan con la obstrucción secundaria del epidídimo (27). En estos casos se inicia el procedimiento liberando los conductos deferentes para poder inyectar a lo largo azul de metileno. El objetivo de esta metodología es poder apreciar la salida de líquido coloreado a través de los meatos eyaculatorios (cromoeyaculoscopia), una vez realizada la resección endoscópica transuretral de los eyaculadores o del posible utriculocele (resección de la cúpula) (28).

De comprobar la ausencia de espermatozoides debe sospecharse la obstrucción secundaria epididimaria. Otra posibilidad es atender al posoperatorio de la cirugía endoscópica. Si se aprecia un aumento del volumen seminal sin la presencia de espermatozoides, ello confirma la obstrucción del tubo epididimario, por lo que deberá procederse a la práctica de una epididimovasostomía. Si se consigue la aparición de espermatozoides en el eyaculado, es aconsejable proceder de inmediato a la criopreservación de los mismos para poder utilizarlos en el futuro en técnicas de reproducción asistida en caso de ser preciso. El motivo de ello es la alta frecuencia con la que se producen reobstrucciones de los conductos eyaculadores (29).

\section{Cirugía cruzada}

Cabe destacar la posibilidad de que exista una obstrucción a cualquier nivel de la vía con una espermatogénesis conservada, una vía seminal contralateral normal y una importante alteración de la espermatogénesis. En estos casos puede aprovecharse la vía conservada para realizar una anastomosis cruzada con la región preobstructiva de la vía con función testicular normal (30). Otro ejemplo de ello es la alteración de la espermatogénesis con normalidad de la vía en un lado y agenesia deferencial contralateral. En este caso puede realizarse una anastomosis cruzada entre el epidídimo agenésico y el deferente contralateral (31).

\section{TÉCNICAS DE RECUPERACIÓN ESPERMÁTICA}

En muchos casos de obstrucción de la vía seminal no es posible realizar técnicas reconstructivas o éstas no resultan exitosas. En estos pacientes es posible obtener espermatozoides al nivel donde se hallen para utilizar en técnicas de reproducción asistida. 


\section{Microaspiración espermática deferencial (MAE-D)}

Cabe destacar la obstrucción distal del conducto deferente y del conducto eyaculador en los que no puede realizarse una cirugía reconstructiva o bien ésta ha fracasado, la eyaculación retrógrada en la que no pueden recuperarse espermatozoides de buena calidad en la orina posorgasmo y la aneyaculación de origen orgánico o psicógeno donde han fracasado las metodologías ensayadas (electrovibración, electroestimulación). En estos casos es posible aspirar espermatozoides del conducto deferente para ser utilizados en técnicas de reproducción asistida (32).

El procedimiento se realiza bajo anestesia local; se libera el conducto deferente y se practica una hemisección transversal hasta apreciar su luz. La micropipeta del mecanismo aspirativo se introduce en el extremo proximal, mientras se realiza la compresión del testículo y el epidídimo.

\section{Microaspiración espermática epididimaria (MAE-E)}

Se halla indicada en aquellos pacientes que presentan una obstrucción epididimaria en los que no es posible la cirugía reconstructiva, como la agenesia bilateral de los conductos deferentes o cuando aquélla ha fracasado. En determinados casos en los que la pareja tiene una edad avanzada puede plantearse como primer acto la aspiración de ovocitos junto a la microaspiración espermática.

Una vez realizada la hiperestimulación ovárica de la pareja, se puncionan los folículos ováricos mediante visión ecográfica transvaginal. Si se obtienen más de tres ovocitos se procede a intervenir al varón, con la misma metodología descrita en la epididimovasostomía. Cuando emana el fluido tubular se comprueba de forma inmediata la presencia de espermatozoides, pasando un portaobjetos por la apertura tubular epididimaria procediendo a observar bajo el microscopio de luz, mientras se observan las características macroscópicas del fluido. Siempre es conveniente aspirar la máxima cantidad posible de espermatozoides con el fin de criopreservarlos y utilizarlos en ciclos posteriores en aquellos casos en los que no se consiga la gestación o cuando la pareja desee tener otro hijo. Ello evita tener que practicar una nueva cirugía.

\section{Microaspiración espermática testicular}

Parte de lo demostrado por algunos autores que indican que con los espermatozoides testiculares se pueden conseguir fertilizaciones y gestaciones (3334). Está indicada en oligozoospermias muy severas, en determinados casos de astenozoospermia total y en azoospermia secretora.

La recuperación espermática se realiza bajo anestesia local, extrayendo uno o varios fragmentos de pulpa testicular que son depositados en un recipiente que contiene el medio de cultivo. En el laboratorio se procede a macerar o disecccionar la muestra entre dos portaobjetos para facilitar el desprendimiento de los espermatozoides.

Cuando los espermatozoides son aspirados del conducto deferente suelen tener una buena calidad, por lo que pueden aplicarse tanto para la ICSI como para la FIV clásica (35-37). Cuando los espermatozoides son aspirados de epidídimo y se han aplicado técnicas de reproducción asistida como FIV clásica y la ICSI, se ha conseguido un $46 \%$ de fertilizaciones mediante la ICSI (7\% FIV clásica) y un 53\%, de gestaciones con la ICSI (9\% FIV clásica) ( 38). Cuando los espermatozoides se obtienen del testículo en casos de azoospermia excretora, el porcentaje de fertilizaciones oscila entre 34 y $62 \%$ y el de gestaciones entre 19 y 57\% (39-41). Cuando se trata de azoospermias secretoras, en aproximadamente el $50 \%$ de los casos se consigue obtener algún espermatozoide para utilizar en la ICSI y los resultados en términos de fertilización oscilan entre el 38 y $58 \%$, y las gestaciones entre el 23 y 43\% (4244).

Hay autores que defienden la punción transescrotal para aspirar espermatozoides del epidídimo o del testículo (45- 46). Este procedimiento tiene algunas críticas: es una técnica que constituye una maniobra a ciegas que puede lesionar de manera excesiva a aquellos órganos, originando hematomas difíciles de controlar y obteniendo un escaso número de espermatozoides para 
criopreservar (47). Los espermatozoides testiculares generalmente son difíciles de localizar_a veces no se hallan_y en un gran número de ocasiones no presentan motilidad. La motilidad es un factor importante para la ICSI teniendo en cuenta que para aplicar la técnica los espermatozoides precisan ser inmovilizados. Ahora bien, en casos de astenozoospermia total, el test hipoosmótico (HOS) u otros tests de vitalidad espermática pueden ser útiles para seleccionar los espermatozoides vivos (48-49).

Por todo ello se cree que la microcirugía sigue siendo la metodología más adecuada para la obtención de espermatozoides deferenciales, epididimarios y testiculares, con miras a una cirugía más limpia, menos traumática y con mayores posibilidades de reintervención.

\section{CIRUGÍA DEL VARICOCELE}

Esta cirugía se halla indicada en aquellos casos de infertilidad en los que existan alteraciones de los parámetros espermáticos (50). El diagnóstico se realiza a partir de la exploración física y del estudio doppler espermático (51).

El tratamiento se basa en seccionar $u$ ocluir las venas espermáticas, fundamentalmente los troncos que confluyen en la vena espermática interna. Algunos autores optan por ligar la totalidad de las venas testiculares, conservando únicamente la vena deferencial (52-53). Entre las muchas técnicas que se han descrito de varicocelectomía cabe destacar la ligadura venosa a diferentes niveles con $y$ sin microcirugía, la embolización espermática y la cirugía laparoscópica.

El abordaje quirúrgico es inguinal o subinguinal, y se puede realizar bajo anestesia local. Esta manera de proceder hace que la varicocelectomía sea un procedimiento sencillo que puede llevarse a cabo de forma ambulatoria. Una vez anestesiada la piel y el cordón espermático a la altura inguinal o subinguinal, se procede a exponer los elementos del condón. Cuando existen troncos venosos de gran calibre, se liberan del tejido periférico y se ligan, intentando siempre respetar la arteria espermática que acostumbra hallarse adherida a la pared posterior del tronco venoso más importante (54).

Bajo microscopio operatorio se identifican correctamente los elementos del cordón, se respetan la arteria espermática y los vasos linfáticos, y se procede a ligar la totalidad de venas por pequeñas que sean. Cuando, a pesar de la magnificación, sea difícil identificar la arteria, es de gran ayuda una gota de papaverina sobre el vaso y observar el latido del mismo (55).

Las complicaciones de la varicocelectomía bajo este abordaje microquirúrgico son poco comunes. La atrofia testicular puede ocurrir si hubo compromiso arterial. El hidrocele ocurre cuando los linfáticos son interrumpidos en su trayecto. El hematoma y la infección de la herida son raros.

\section{AGRADECIMIENTOS}

Al doctor Jaime Saavedra Saavedra, por su contribución con la revisión y asesoría de este trabajo.

\section{REFERENCIAS BIBLIOGRÁFICAS}

1. Pomerol JM. Práctica microquirúrgica. En: Práctica andrológica. Pomerol JM, Arrondo JL, editores. Barcelona, Masson-Salvat; 1994. p. 282-98.

2. World health Organization. Laboratory manual for the examination of human semen and sperm-cervical mocus interaction. 3a. ed. Cambridge: Cambridge University Press; 1992.

3. Goldstein M. Vasography. En: Goldstein M. Surgery of male infertility. Philadelphia: W.B. Saunders Company; 1995. p. 26-31.

4. Ruiz-Romero J, Narváez H, Bassas L, Pomerol JM. Consideraciones sobre la relación entre la agenesia de conductos deferentes y fibrosis quística. Ac Func Puigvert 1993;12:236-40.

5. Casals T, Bassas L, Ruiz Romero J et al. Extensive analysis of 40 infertile patients with congenital absence of the vas deferens: in $50 \%$ of cases only CFTR allele could be detected. Hum Genet 1995;95:205-11.

6. Balaban B, Urman B, Sertac A, Alatas C, Aksoy S, Mercan R, Nuhoglu A. In-vitro culture of spermatozoa induces motility and increases implantation and pregnancy rates after testicular sperm extraction and intracytoplasmic sperm injection. Hum Reprod 1999;14:2808-11.

7. Palermo GD, Schlegel P, Hariprashad JJ, Ergun B, Mielnik A, Zaninovic N. Fertilization and pregnancy outcome with intracytoplasmic sperm injection for azoospermic men. Hum Reprod 1999;14:741-8.

8. Murphy H, George C, Kretser D, Judd S. Successful treatment with ICSI of infertility caused by azoospermia associated with adrenal restts in the testes. Hum Reprod 2001;2:263-7.

9. Narváez H, Saavedra J. Papel del andrólogo en la salud reproductiva. Rev Colomb Obstet Ginecol 2000;51:105-11. 10. Goldstein M. Microsurgical vasoepididymostomy end-toend anastomosis. En: Goldstein M. Surgery of male infertility. Philadelphia: W.B. Saunders Company; 1995. p. 120-27. 
11. Pomerol JM. Microcirugía del epidídimo. En: Pomerol JM, Arrondo JL. Práctica Andrológica. Barcelona: Masson-Salvat; 1994. p. 299-318.

12. Silber SJ. Microscopic vasoepididymostomy: specific microanastomosis to the epididymal tubule. Fertil Steril 1978;30:565.

13. Schlegel PN, Goldstein M. Microsurgical vasoepididymostomy: refinements and results. J Urol 1993;150:1165-8.

14. Matsuda T, Horii Y, Muguruma K, et al. Microsurgical epididymovasostomy for obstructive azoospermia: factors affecting postoperative fertility. Eur Urol 1994;26:322-6.

15. Silber SJ. Results of microsurgical vasoepididymostomy: role of epididymis in sperm maturation. Hum Reprod 1989;4:298.

16. Fuchs EF. Restoring fertility through epididymovasostomy. Comtemp Urol 1991;3(12):27.

17. Derrick FC, Yarbrongn W, D’Agostino J. Vasovasostomy: results of questionnaire of members of the American Urological Association. J Urol 1973;110:556.

18. Belker AM, Konnak JW, Sharlip ID, Thomas AJ. Jr. Intraoperative observations during vasovasostomy in 334 patients. J Urol 1983;129:524.

19. Silber SJ. Vasectomy and vasectomy reversal. Fertil Steril 1978;29:125-40.

20. Goldstein M. Vasectomy and vasectomy reversal. Curr Ther Endocrinol Metab 1985-1986;1986:180-5.

21. Belker AM, Fuchs EF, Konnak JW et al. Transient fertility after vasovasostomy in 892 patients. J Urol 1985;134:75.

22. Linnet L. Clinical immunology of vasectomy and vasovasostomy. Urology 1983;22:101-14.

23. Belker AM, Thomas AJ. Jr, Fuchs EF et al. Results of 1469 microsurgical vasectomy reversals by the Vasovasostomy Study Group. J Urol 1991;145:505-11.

24. Silber SJ. Pregnancy after vasovasostomy for vasectomy reversal: a study of factors affecting long-term return of fertility in 282 patients followed for 10 years. Hum Reprod 1989;4:318-22.

25. Matsuda T, Horii Y, Yoshida O. Unilateral obstruction of the vas deferens caused by childhood inguinal herniorhaphy in male infertility patients. Fertil Steril 1992;58:609-13.

26. Belker AM, Jiménez-Cruz FJ, Kêlami A, Wagenknecht LV. Alloplastic spermatocele: poor sperm motility in intraoperative epididymal fluid contraindicates prosthesis implantation. J Urol 1986;136:408.

27. Vicente J, Del Portillo L, Pomerol JM. Endoscopic surgery duct obstruction of the ejaculatory ducts. Eur Urol 1983;9:338. 28. Hendry WA, Prior JP. Mullerian duct (prostatic utricle) cyst: diagnosis and treatment in subfertile males. Br J Urol 1992; 69:79-82.

29. Pomerol JM, Enrique C, Narváez H, Puigvert A. Asthenonecrozoospermia and hypospermia as indicative pathology of the distal seminal tract. Int $\mathrm{J}$ Androl 1997;20(suppl):42.

30. Lizza EF, Marmar JL, Schmidt SS, et al. Transseptal crossed vasovasostomy. J Urol 1985;134:1132-4.

31. Sabanegh Jr. E, Thomas AJ Jr. Effectiveness of crossover transseptal vasoepididymostomy in treating complex obstructive azoospermia. Fertil Steril 1995;63:392-5.

32. Narváez $H$, Navas $M$, Viscasillas $P$, Pomerol JM. Microaspiración de espermatozoides a nivel deferencial y fertilización in vitro. En: Libro de resúmenes VII Congreso Nacional de Andrología. ASESA. Bilbao, España; 1995. p. 526-27.

33. Silber SJ, Van Steirteghem AC, Liu J, et al. High fertilization and pregnancy rate after intracytoplasmatic sperm injection with spermatozoa obtained from testicle biopsy. Hum Reprod 1995;10:148-52.

34. Schoysman R, Vanderzwalmen P, Nijs M et al. Pregnancy after fertilization with human testicular spermatozoa. Lancet 1993;342:1237.

35. Pryor J, Parsons J, Goswamly R et al. In vitro fertilization for men with obstructive azoospermia. Lancet 1984:762
36. Pomerol JM, García F, Barri PN y cols. Gestación mediante esperamatozoides aspirados del conducto deferente y fertilización in vitro. Arch Esp Uro 1993;45:811-5.

37. Temple-Smith PD, Southwick GJ, Yates CA, et al. Human pregnancy by in vitro fertilization using sperm aspirated from the epididymis. J In Vitro Fert Embryo Transfer 1985;2:119.

38. Silber SJ, Nagy ZP, Lui J. Conventional in-vitro fertilization versus intracytoplasmatic sperm injection for patients requiring microsurgical sperm aspiration. Hum Reprod 1994;9:1705-9.

39. Hovatta O, Moilanen J, Von Smitten K, et al. Testicular needle biopsy open biopsy epididymal aspiration and intracytoplasmatic sperm injection in obstructive azoospermia. Hum Rep 1995;10:2595-9.

40. Gil-Salom M, Mínguez Y, Rubio C, et al. Efficacy of intracytoplasmatic sperm injection using testicular spermatozoa. Hum Reprod 1995;10:3166-70.

41. Mansour R, Aboulghar M, Serour G, et al. Intracytoplasmatic sperm injection using microsurgically retrieved epididymal and testicular sperm. Fertil Steril 1996;65:566-72.

42. Tournaye H, Liu J, Nagy PZ, et al. Correlation between testicular histology and outcome after intracytoplasmatic sperm injection using testicular spermatozoa. Hum Reprod 1996;11:127-32.

43. Devroey P, Nagy Z, Tournaye H, et al. Outcome of intracytoplasmatic sperm injection with testicular spermatozoa in obstructive and non-obstructive azoospermia. Hum Reprod 1996;11:1015-8.

44. Kahraman S, Ozgür S, Alatas C, et al. Fertility with testicular sperm extraction and intracytoplasmatic sperm injection in non-obstructive azoospermic men. Hum Reprod 1996;11:756-60.

45. Craft IL, Khalifa I, Boulos A, et al. Factors influencing the outcome of in-vitro fertilization with percutaneous aspirated epididymal spermatozoa and ICSI in azoospermic men. Hum Reprod 1995;10:1791-4.

46. Shrivastav P, Nadkarni P, Wensvoort S, Craft I. Percutaneous epididymal sperm aspiration for obstructive azoospermia. Hum Reprod 1994;9:2058-61.

47. Shufaro Y, Prus D, Laufer N, Simon A. Impact of repeated fine needle aspirations (TEFNA) and testicular sperm extraction (TESE) on the microscopic morphology of the testis: an animal model. Hum Reprod 2001;16:11.

48. Nijs M, Vanderzwalmen P, Vandamme B, Segal-Bertin G, Lejeune B, Segal L, Van Roosendaal E, Schoysman R. Fertilizing ability of immotile spermatozoa after intracytoplasmatic sperm injection. Hum Reprod 1996;11:2180-5

49. Sallam HN, Farrag A, Agameya AF, Ezzeldin F, Eid A and Sallam A. The use a motified hypo-osmotic swelling test for the selection of viable ejaculated and testicular immotile spermatozoa in ICSI. Hum Reprod 2001;16:272-6.

50 . Word Health Organization. The influence of varicocele on parameters of fertility in a large group of men presenting to infertility clinics. Fertil Steril 1992;57(4):725.

51. Bast FA, Masabni R. Effective of varicocelectomy in varicoceles diagnosed by physical examination versus doppler studies. Fertil Steril 1988;50(2):321.

52. Palomo A. A radical cure of varicocele by a new technique. J Urol 1949;61:604-7

53. Dubin L, Amelar RD. Varicocelectomy: 986 cases in a 12 year study. J Urol 1977;10:446-9.

54. Marmar IL, Kym Y. Subinguinal microsurgical varicocelectomy: A technical critique and statiscal analysis of semen and pregnancy data. J Urol 1994;152:1127.

55. Goldstein M, Gilbert BR, Dicker A, Dúos J, Gnecco C. Microsurgical inguinal varicocelectomy with delivery of the testis: An artery and lymphatic sparing technique. J Urol 1992;148(6):1808-11. 